University of Wollongong

Research Online

Australian Institute for Innovative Materials -

Papers

Australian Institute for Innovative Materials

$1-1-2018$

Quantitative characterisation of conductive fibers by capacitive coupling

Andres Ruland Palaia

University of Wollongong, ruland@uow.edu.au

Rouhollah Jalili

University of Wollongong, rjalili@uow.edu.au

Attila J. Mozer

University of Wollongong, attila@uow.edu.au

Gordon G. Wallace

University of Wollongong, gwallace@uow.edu.au

Follow this and additional works at: https://ro.uow.edu.au/aiimpapers

Part of the Engineering Commons, and the Physical Sciences and Mathematics Commons

Research Online is the open access institutional repository for the University of Wollongong. For further information contact the UOW Library: research-pubs@uow.edu.au 


\title{
Quantitative characterisation of conductive fibers by capacitive coupling
}

\begin{abstract}
This work presents a study on a capacitively coupled contactless conductivity detector (C4D) for micronsized fibers. Following a previous report on the qualitative application of $C 4 D$ for fibers, the present study provides a thorough analysis of the signal response to fiber conductivity. Using reduced graphene oxide (RGO) fibers, the detector response as a function of fiber length, cross-sectional area and resistance has been investigated. To study the effect of insulating coatings, Parylene-coated RGO fibers were also investigated. In addition, measurements were performed in different coupling environments, such as in a capillary tube or air. The analysis of the measured data allowed the determination of the C4D conductivity of various RGO fibers, and the correlation with contact methods through empirical relationships to be determined. It was found that the detection limit and sensitivity of resistance measurements are mainly dependent on the sensor design, and also on the fiber properties. The detection threshold can be defined as the ratio of the coupling impedance to fiber resistance. In our case, the detection limit was found for impedance ratios equal to 14. This limit sets a functioning mode in C4D for fibers, which may be used as an area or resistance detector for the impedance ratio above or below the detection threshold. A semi-log linear response of the fiber resistance to the voltage output was found for impedance ratios between 2.66 and 0.63 . These impedance ratios may serve as a reference for designing C4D, depending on the fibers to be tested and the analytical information needed. In summary, we suggest that C4D has the capacity to emerge as a new characterisation tool for micron-sized fibers, due to its applicability to any conductive material, ease of use, and the contactless nature of the measurement.
\end{abstract}

\section{Disciplines}

Engineering | Physical Sciences and Mathematics

\section{Publication Details}

Ruland, A., Jalili, R., Mozer, A. J. \& Wallace, G. G. (2018). Quantitative characterisation of conductive fibers by capacitive coupling. The Analyst, 143 (1), 215-223. 


\title{
Quantitative Characterisation of Conductive Fibers by Capacitive Coupling
}

Andres Ruland ${ }^{\mathrm{a}}$, Rouhollah Jalili ${ }^{\mathrm{b}}$, Attila J. Mozer ${ }^{\mathrm{a}}$, and Gordon G. Wallace ${ }^{\mathrm{a}^{*}}$

a ARC Centre of Excellence for Electromaterials Science (ACES), Intelligent Polymer Research Institute, AlIM Facility, Innovation Campus, University of Wollongong, NSW 2500, Australia. E-mail: gwallace@uow.edu.au

${ }^{\mathrm{b}}$ School of Engineering, College of Science, Engineering \& Health, RMIT University, Melbourne, VIC 3001, Australia.

\begin{abstract}
This work presents a study on a Capacitively Coupled Contactless Conductivity Detector (C4D) for micron-size fibers. Following a previous report on the qualitative application of C4D for fibers, the present study provides a thorough analysis of the signal response to fiber conductivity. Using reduced graphene oxide (RGO) fibers the detector response as a function of fiber length, crosssection area and resistance has been investigated. To study the effect of insulating coatings, Parylene coated RGO fibers were also investigated. In addition, measurements were performed in different coupling environments, such as an in a capillary tube or air. Analysis of the measured data allowed the determination of C4D conductivity of various RGO fibers, and the correlation to contact methods through empirical relationships to be determined. It was found that the detection limit and sensitivity of resistance measurements is mainly dependent on the sensor design, but also on the fiber properties. The detection threshold can be defined as the ratio of coupling impedance to fiber resistance. In our case, detection limit was found for impedance ratios equal to 14 . This limit sets a functioning mode in C4D for fibers, which may be used as an area or resistance detector for impedance ratio above or below the detection threshold. A semi-log linear response of fiber resistance to voltage output was found for impedance ratios between 2.66 and 0.63 . These impedance ratios may serve as a reference for designing C4D for depending on the fibers to be tested and the analytical information needed. In summary, we suggest that C4D has the capacity to emerge as a new characterisation tool for micron-size fibers, due to its applicability to any conductive material, ease of use, and the contactless nature of the measurement.
\end{abstract}

\section{Introduction}

In recent years, a great deal of attention has been paid to into the development of electrically conductive organic fibers as an alternative to metal wires. Among them, reduced graphene oxide 
(RGO), RGO-polymer composites and carbon fibers are some of the best candidates. ${ }^{1,2}$ These new types of fibers have the benefit of good conductive properties combined with lightweight, as well as mechanical strength, flexibility and cost-effective manufacturing. Such fibers can be further assembled into more complex structures such as yarns, ${ }^{3,4}$ or knitted into textiles. ${ }^{5}$ Applications include the development of smart textiles, wearable electronics, or artificial muscles among others. ${ }^{6-11}$ While there is intense research into such applications, there are fewer reports of methods for fiber or yarn characterisation. Until now, the main tools used for evaluating the fiber resistance are contact methods, such as the two- or four-point point probe (2PP and 4PP, respectively) methods. In addition, to evaluate the fiber resistance per area, resistivity, cross-section areas must be known in advance. This is typically done by microscopy, which are time consuming and difficult to implement to simultaneous resistance measurements. For in-situ cross-section area determination, laser based systems have been developed, but are limited to circular fibers. Concerning resistance measurements, use of mechanical pressure from electrode to fiber, or use of silver paste, render the fiber unusable. Hence, it can be concluded that contact methods are time consuming and, overall, of destructive character.

An effort towards continuous fiber resistance monitoring of conductive fibers was developed by Bashir et al. $^{12}$ In that work, fiber resistance was monitored by 2PP and 4PP, where brass pulleys acted as both sample holder and electrode. Although effective for monitoring conductive fibers, the method is impractical for enamelled or coaxial fibers, or measurements requiring a contactless approach. For instance, for in-situ monitoring of GO reduction using light induced reduction, ${ }^{13,14}$ chemicals, ${ }^{15}$ or after heat treatment. ${ }^{16,17}$

In regard to contactless conductivity measurements, a few other techniques based on microwave and radiofrequency methods are available, such as microwave cavity perturbation and Eddy Current. In the case of microwave conductivity, most reports focus on thin film characterisation, and only a few examples on thin fibers exist. ${ }^{18,19}$ Despite being effective for measuring conductive films at high throughput, it requires a complex setup such as a special resonance cavity design capable of operating at $\mathrm{GHz}$ frequencies. ${ }^{20,21}$ On the other hand, Eddy Current is a well stablished technique in the field of non-destructive testing, and it is commercially available for measuring various forms of test samples. One of the limitations of Eddy Current is the need of a test sample with sufficient volume in order to induce detectable changes. This limits its applicability to large objects, or 2D structures such as foils or textiles, and thick wires (>1 mm). 
Recently, Cabot et al., ${ }^{22}$ demonstrated the possibility to qualitatively evaluate the conductive properties of micron-size fibers in a contactless fashion. In particular, by using a Capacitively Coupled Contactless Conductivity Detector (C4D). C4D is a commonly used technique in the field of separation science / electrofluidics, ${ }^{23-25}$ as an analyte detector in capillary electrophoresis. However, its application to conductive fibers is rather novel and not fully understood. Open questions are for instance, how the signal is influenced by the fiber's cross-section area, the detection limit, and if it can be used to quantify conductivity of micron-sized fibers. In the present work, we have performed a thorough study on signal response to different fiber characteristics, with the aim of extracting quantitative information on fiber's properties from C4D signal output. In particular, by monitoring C4D signal output as a function fiber length, area and resistance. Quantitative evaluation of fibers properties was based on parametric analysis, empirical models and their validation with experimental measurements. Limitations and possibilities of the method are also discussed throughout the text.

\section{Working principle of C4D for conductive fibers}

The working principle of C4D is a well-established concept, intensively used since 1998 as analyte detection in capillary electrophoresis. ${ }^{26,27} \mathrm{~A}$ detailed description of its fundamentals and the developments of the technique can be found elsewhere. ${ }^{28}$ Briefly, two tubular electrodes, emitter and receiver, are isolated from each other by a gap, typically defining the detection section. In our case, the electrode width and gap are 0.5 and $1 \mathrm{~cm}$, respectively (Figure $1 \mathrm{~d}$ ).

a)

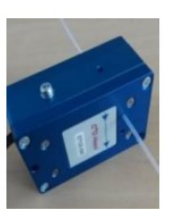

b)

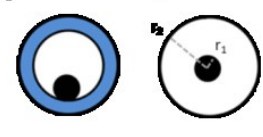

d)

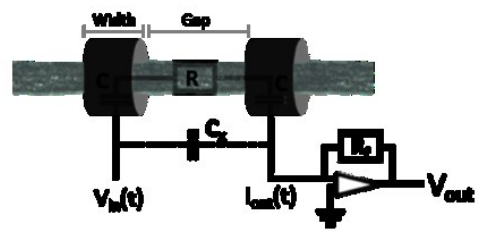

Figure 1: a) Photograph of the C4D sensor used in this work, fitted with a capillary tube. b, c) Schematic representation of a cross-section view of a conductive fiber with respect to tubular electrodes, (b) off-centre and using a capillary tube, (c) and centred with air as dielectric medium . Electrode and fiber radii are represented as $r_{2}$ and $r_{1}$, respectively. d) Equivalent circuit diagram of the sensor superimposed to actual interfaces in fiber measurement. Grounded shield not included for clarity. C: coupling capacitance, $C_{x}$ : stray capacitance, $R$ : fiber resistance, $R_{f}$ : Feedback resistor, $V_{\text {in }}$ $(t)$ : applied AC-voltage at emitter electrode, $\mathrm{I}_{\text {out }}(\mathrm{t})$ : collected $\mathrm{AC}$-current arriving at the receiver 
electrode, $\mathrm{V}_{\text {out }}$ : peak voltage of the resulting AC-Voltage after current to voltage conversion and amplification. Note that electrode radius to fiber are not on scale.

The sample under examination (e.g. fluid or conductive fiber) is physically separated from the electrodes, typically by a thin dielectric medium or a capillary tube. After applying an AC-voltage, $\mathrm{V}_{\text {in }}$, to the emitter electrode, the dielectric medium near the electrode is capacitively charged. The induced charge flows through the detection gap as a function of the sample resistance, R, (e.g. conductive fiber) and reaches the receiver electrode area via an AC potential difference. The resulting AC-current travels through the dielectric medium to the receiver electrode. Finally, the current is amplified by a feedback resistor, $R_{f}$ and converted back to AC-voltage. The nominal signal output, $V_{\text {out, }}$ is the peak value of the resulting AC-voltage. Crucial for this process is the need for sufficiently high excitation frequency to overcome the capacitive impedance, $Z_{c}$, between electrode and sample. Likewise, a thin dielectric material is used to lower $Z_{c}$. The amplitude of $V_{\text {out }}$ can be tuned, in addition to $Z_{c}$, by varying $V_{\text {in }}$ and $R_{f}$. $A$ short path for the above mentioned mechanism is stray capacitance, $C_{x}$, which is the result of direct coupling through air between both electrodes. In our study, $C_{x}$ is neglected since the electrodes are shielded. The remaining voltage from stray capacitance is set to zero before each measurement.

For quantitative analysis, $\mathrm{V}_{\text {out }}$ was converted to impedance variation, $\Delta \mathrm{Z}$, using equation (1).

$$
\Delta Z=\frac{V_{\text {in }} R_{f}}{V_{\text {out }}}(1)
$$

Since impedance variation is due only to current flow through a capacitance-resistor-capacitance circuit, $\Delta Z$ is equal to fiber resistance and twice the coupling impedance, $Z_{c}$ (equation 2 ).

$$
\Delta Z=R+2 Z_{c}
$$

$Z_{c}$ is inversely proportional to the coupling capacitance, $C_{c}$, and frequency, $f$, as shown in (equation 3). As coupling capacitance (equation 4), it is assumed a double cylinder capacitor, of width $w$. The outer and inner radii of the cylinder, which defines the dielectric thickness, are $r_{2}$ and $r_{1}$, respectively. Note that in Figure $1 c, r_{2}$ and $r_{1}$ correspond to electrode and fiber radii, respectively. $\varepsilon_{r}$ and $\varepsilon_{0}$ are the relative and absolute dielectric constant of the coupling media and vacuum, respectively.

$$
\begin{gathered}
Z_{c}=i \frac{1}{2 \pi f C_{c}}(3) \\
C_{c}=\frac{2 \pi \varepsilon_{o} \varepsilon_{r} w}{\operatorname{Ln}\left(r_{2} / r_{1}\right)}
\end{gathered}
$$


Finally, with known values of $\Delta Z$ and $Z_{c}$, detection gap, I $(\mathrm{cm})$, and sample area, $A\left(\mathrm{~cm}^{2}\right)$, conductivity, $\mathrm{K}(\mathrm{S} / \mathrm{cm})$ can be calculated by equation 5 . Same equation applies for 4PP conductivity.

$$
K=\frac{1}{R} \frac{l}{A}(5)
$$

\section{Experimental}

\section{Fiber preparation}

The fibers were prepared by a wet-spinning method following a previously reported procedure. ${ }^{17}$ Briefly, graphene oxide (GO) in liquid crystal phase at a concentration of $10.5 \mathrm{mg} / \mathrm{ml}$ was injected into a rotating coagulation bath at $3 \mathrm{~cm}$ from the center. Composition of the coagulation bath was: $70 \%$ Ethanol, $25 \%$ Water, $5 \%$ and Hypophosporous acid (HA) as reducing agent - percentages are expressed in volume \%. $\mathrm{CaCl}_{2} 2 \%$ in weight was also added. GO fibers reduction was carried out by placing the GO-fibers-coagulation bath in an oven at $80^{\circ} \mathrm{C}$ overnight. Some fibers were collected at particular times to monitor fiber resistance. The coagulation solution was removed from the resulting RGO fibers and washed 3 times in deionised water until neutral pH. For the fiber F1, same procedure was repeated but with a GO of $20 \mathrm{mg} / \mathrm{ml}$. To achieve different cross-sectional areas, fibers were spun using a needle gauge 19 (fibers 1-3) and gauge 21 (fibers 4-6). In addition, fibers were tensioned under different weights: $1 \mathrm{~g}$ (Fiber 1, 2, 4), $2 \mathrm{~g}$ (Fiber 5) and $2.8 \mathrm{~g}$ (Fiber 3, 6). Stainless steel fiber 316L purchased from Goodfellow Cambridge Ltd. Chemical used: HA 50 wt \% and $\mathrm{CaCl}_{2}$ purchased from Sigma-Aldrich. Ethanol purchased from Chem-supply. For Parylene coating, Parylene type C was deposited with a coater from Speciality Coating Systems PDS 2010.

\section{Fiber characterisation}

RGO fibers were characterised with an in-house 4PP setup connected to a multimeter (Keithley 2000). Note that no silver paste was used. For a robust electrical contact, the fiber was gently pressed in between a soft block and the electrode tips $(0.23 \mathrm{~cm}$ spacing). Measurements were repeated 3 times on independent fiber fragments. For cross-sectional area measurements, an optical microscope (Leica DM6000) was used. For that, fibers were first encapsulated into polymer matrix via mild heat polymerisation ( $80 \mathrm{C}$ overnight) of a monomer, and then polished orthogonally to fiber length (Leica EM TXP). Average cross-section areas were taken from 5 independent fragments via image analysis of the corresponding optical images. The C4D setup (EDAQ ET125) consisted of a detector of $1 \mathrm{~cm}$ electrode gap (instead of $9 \mathrm{~mm}$ as specified by manufacturer), $5 \mathrm{~mm}$ electrode width, $1.6 \mathrm{~mm}$ aperture, variable AC-frequency $(20 \mathrm{KHz}-2 \mathrm{MHz})$ and variable peak-to-peak input voltage (2-20 V). Two different feedback resistances, $R_{f}$, were used, i.e. 11 and $56 \mathrm{kOhm}$. The input voltage was adjusted to avoid signal output above $2.5 \mathrm{~V}$. Displayed signals over $2.5 \mathrm{~V}$ were recorded at lower input voltages, and rescaled to $20 \mathrm{~V}$ for comparison. As capillary tube, a PTFE tube of 1.6 
and $0.8 \mathrm{~mm} \mathrm{OD/ID}$ was used. Voltage values recorded for measurements inside the capillary tube corresponded to 3 reproducible maximum voltage values. For measurements using a capillary, the fiber position respect to the electrodes was off-centre as in figure $1 \mathrm{~b}$. The fibers did not move during measurements as the capillary was placed horizontally. In addition, there was a strong surface affinity between PTFE and fibers, facilitating a static position of the fiber inside the capillary. For air measurements, fiber was guided though a homemade holder featuring a fiber guide aligned to each aperture. Before recording the signals, the guide was adjusted until the fiber was at the center of electrode aperture - corresponding to the minimum voltage output. Measurement details in all cases, unless specified, were: Voltage: $20 \mathrm{~V}$ pp, Frequency: $2 \mathrm{MHz}$, amplification: $11 \mathrm{kOhm}$ (for capillary) and $56 \mathrm{kOhm}$ (for air). Stray voltage was set to zero before each measurement.

\section{Results and discussion}

\section{Signal dependence on fiber length, resistance and area}

Before using C4D as a quantitative conductivity detector, a study of the signal output as a function of fiber characteristics was first carried out. Due to its analogy to traditional C4D, most of the experiments shown were conducted using a capillary tube, as the coupling medium and the sample holder. For comparative purposes, additional measurements were carried out using air as coupling medium (Figures $1 b$ and $1 c$, respectively). Air measurements also represent the ideal setup for contactless characterisation, suitable for instance for industrial scale fiber monitoring. In a previous work, it was concluded that the signal output can be scaled to 4PP fiber resistance and influenced by its cross-sectional area ${ }^{22}$, termed hereafter as area. In this case we shine more light into the underlying mechanism governing capacitive conductivity for micron-sized fibers, by analysing the signal output in terms of fiber's area, length and resistance. The fibers studied in this work and their relevant parameters are listed in Table 1. See experimental section for details. Representative crosssectional images for each fiber are shown in Figure S1. 
Table1. List of fibers studied in this work.

\begin{tabular}{|c|c|c|c|c|}
\hline Sample name & Resistance ${ }^{+}(\mathbf{O h m s ~} \mathbf{~ c m})$ & Area $^{*}\left(\mathbf{1 0 ^ { - 5 }} \mathbf{c m}^{\mathbf{2}}\right)$ & Conductivity (S/cm) $^{\text {Voltage }}{ }^{\#}$ (V) \\
\hline SS & $23.9 \pm 2$ & $0.31 \pm 0.01$ & $1.34 \mathrm{E} 4$ & - \\
\hline F1 & $426 \pm 5$ & $3.90 \pm 0.06$ & 60.2 & 2.05 \\
\hline F2 & $640 \pm 28$ & $2.53 \pm 0.05$ & 61.6 & 1.94 \\
\hline F4 & $840 \pm 18$ & $1.83 \pm 0.03$ & 64.9 & 1.83 \\
\hline F5 & $1052 \pm 23$ & $1.41 \pm 0.09$ & 67.4 & 1.76 \\
\hline F6 & $1771 \pm 21$ & $1.06 \pm 0.04$ & 73.8 & 1.63 \\
\hline
\end{tabular}

SS: Stainless steel fiber. F1-F6: Reduced graphene oxide fibers. +: Average of 3 independent fragments, *: Average of 5 independent fragments, \#: Voltage output corresponding to 3 reproducible maxima, recorded on $2 \mathrm{~cm}$ fiber length.

The voltage response to different fiber lengths was first examined. To also evaluate the effect of fiber area, fibers F2 and F5 were compared. In addition, different volumes of distilled water were also measured for comparison as high resistance samples. Note that sample length equals to a different longitudinal length of sample inside the capillary. Each data point corresponds to a sample of different length. For fragments shorter than the sensor casing, the capillary was moved until maximum signal was achieved. The displacement of the capillary served as an indication to locate the position of the fiber respect to the electrodes, which was qualitatively at the center of the electrode gap. For capillary measurements, only straight fibers with full contact to the capillary tube were used, as air gap in between them strongly influences signal output. ${ }^{22}$ Likewise, due to the flexible nature of the capillary used, before recording each data point, the capillary was finely adjusted until a maximum voltage output was achieved. The resulting voltage output, $v_{\text {out, }}$ as a function of sample length is shown in Figure 2a, and corresponds to 3 reproducible maximum voltages. The solid lines are an eye guide for connecting experimental data points. 
a)

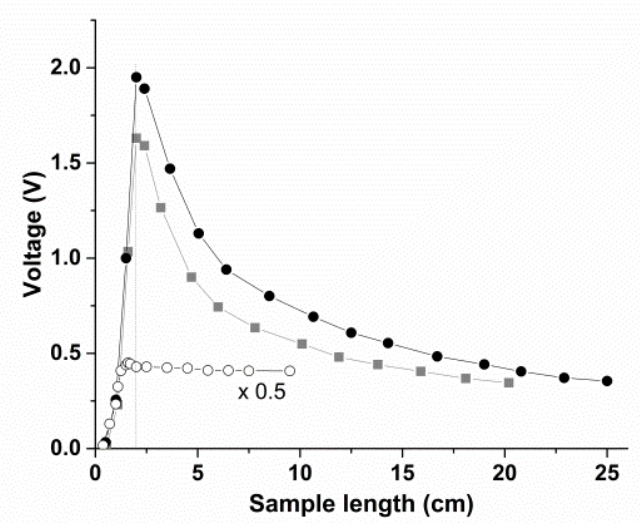

b)

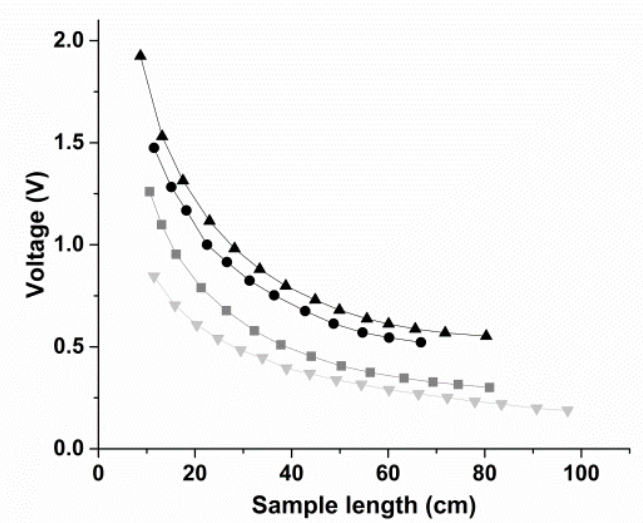

Figure 2. Voltage output as a function of sample length measured in a capillary tube (a) and in air (b). (a) Data is represented for F2 (dots), F5 (squares) and distilled water (open circles). Distilled water data is divided by a factor of 2 for clarity. (b) In addition to (a), data represented for F1 (upper triangles), and SS (lower triangles). Lines between experimental points and vertical mark $2 \mathrm{~cm}$ are added as an eye guide.

Comparing the trends for distilled water and fibers, the data represented in Figure 2a clearly indicates different behaviour depending on the sample properties. Although the comparison is based on two samples very different in nature, the signal only concerns with impedance variations occurring in between the electrodes. Impedance variations are due to changes in the coupling capacitance and resistance, as shown in equation 2 .

For fibers, to highlight is the sharp voltage maximum at $2 \mathrm{~cm}$, and the gradual signal decay beyond that point. In contrast, distilled water shows a gradual increase until $1.6 \mathrm{~cm}$, where it reaches a nearly constant value. The location of the maximum voltage in terms of length illustrates the effective detection gap. For fibers, the effective gap is located at $2 \mathrm{~cm}$, corresponding to the electrode gap and twice the electrode width. Note that for distilled water, an effective gap of $1.6 \mathrm{~cm}$ is slightly larger than the expected. Considering that electrode gap is $1 \mathrm{~cm}$, an increase $0.3 \mathrm{~cm}$ of the detection gap into each electrode occurs. Typically, the increased detection gap in low conductivity solutions is on the sub-millimetre range, for the excitation frequency used. ${ }^{23,25}$ The wider detection gap observed for water is attributed to the dimensions of the capillary tube used, which is thicker and with larger external diameter to traditional ones. The observed difference in detection gap of fibers respect to distilled water is in agreement as previously mentioned, ${ }^{29}$ where low resistance samples cause electric field to concentrate only in the area below electrodes. As a result, detection 
gap for fibers includes the electrode gap and twice the electrode width. For higher resistance samples, electric field coupling is less intense and delocalised towards the electrode gap.

In relation to the voltage decay as a function of sample length, it appears to be related to sample resistance, as it does not occur for distilled water. The hypothesis is that capacitive charge accumulated on the fiber tends to dissipate along its whole length, while for distilled water, due to its high resistance, remains nearly constant. The next observable difference in Figure $2 \mathrm{a}$ is the voltage amplitude. Comparing Fibers F2 and F5, the voltage signal must be related to differences in area, as F2 is larger than F5. However, the ratio between voltages is not proportional to that of the areas. When normalising the voltage profiles for F2 and F5, a slight difference on the amplitudes can be seen in between 5 and $10 \mathrm{~cm}$ of fiber length (Figure 2Sa). The fitting functions and their average are shown in Figure S2b. Despite the slight variation in the range from 5 to $10 \mathrm{~cm}$, the averaged fitting function could be used to extrapolate the voltage at $2 \mathrm{~cm}$ for any fiber length measured on this range. The effect of the resistance does not seem to have a quantitative effect on signal output for fibers F2 and F5. On the other hand, distilled water sample shows a reduced signal output, for an area of about 500 times larger than the fibers. Here the low output signal is due to the large resistance, and a corresponding low impedance change (equation 1). These observations indicate that there must be a resistance detection limit where only the changes in area are detectable.

A similar study was done for air as the coupling medium between the fiber and the electrode. Due to practical limitations, minimum sample lengths were restricted to about $8 \mathrm{~cm}$. This setup also allowed the evaluation of the signal output for longer fiber lengths. In this case, samples up to about 1 meter were used. To gain further information on the voltage signal, a stainless steel fiber (SS) was compared to Fibers F1, F2 and F5. The resulting data is presented in Figure $2 \mathrm{~b}$.

When analysing the voltage decay trends, all fibers adjust to a bi-exponential function $\left(R^{2}=0.99\right)$, with constant voltage values at long fiber lengths. The fitting equation was (equation 6):

$y=A_{1} \exp \left(-x / t_{1}\right)+A_{2} \exp \left(-x / t_{2}\right)+y_{0}(6)$

This bi-exponential dependence suggests that signal decay is proportional to sample area and resistance. Due to the signal decay, it was found that meaningful data could only be extracted from the offset values $\left(y_{0}\right)$ resulting from the fitting functions. These values should also simulate measurements performed on infinite long fibers. These extracted onset values were $0.506,0.420$, $0.265,0.097 \vee$ for F1, F2, F5, and SS, respectively. See Table S1 for details on fitting coefficients. 
Similar as mentioned above, voltage values follow the same trend to the fiber's area. No particular trend was observed on the other fitting coefficients.

To further study the relationship between fiber area and voltage, additional measurements were carried out. F5 fibers were segmented in equal lengths of $2 \mathrm{~cm}$, and the signal response was measured as a function of the number of fragments enclosed inside a capillary tube. Care was taken to ensure superposition of fiber fragments during measurements. The resulting plot (Figure S3) shows an exponential increase of voltage as a function number of fibers, which tend towards a plateau.

To assess the sensor's detection limit, fibers from batch F2 at different reduction times where measured in a capillary tube. The resulting measured voltage as a function of reduction time for fibers of $2 \mathrm{~cm}$ in length is shown in Figure 3. In addition, the 4PP resistance of each fiber is represented on a logarithmic scale due to the large variation on resistances. Voltage variation occurs from about 1 to $2 \mathrm{~V}$, for a GO fiber and RGO, respectively. This voltage change should in principle represent a resistance variation from the MOhm range to a few $\mathrm{kOhm}$. For initial reduction stages, there is a good correlation between voltage and fiber resistance. In contrast, further reduction periods do not seem to follow a proportional voltage increase, as the fiber resistance is further reduced. By performing a fitting function to the voltage as a function of time (data not shown), signal reaches a plateau after 3-4 hours of reduction time. This implies that the resistance detection limit is about $7 \mathrm{kOhm} \cdot \mathrm{cm}$ for this particular sensor. Therefore, observed changes in signal amplitude for fibers below such threshold are due variations in fiber's cross-sectional area. This is consistent with the data on observation in Figure 2. Note that sample after 10 hours reduction corresponds to a tensioned fiber, while the other fibers are non-tensioned. Non-tensioned fibers have typically a rougher surface and increased outer area respect to tensioned ones, affecting the coupling, and in turn, resulting in higher voltage. 


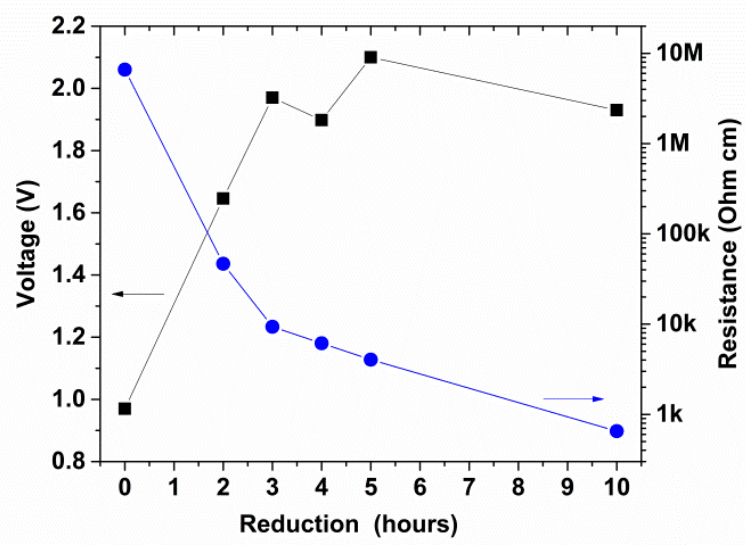

Figure 3. Voltage dependence of $\mathrm{GO}$ fibers to different reduction times at $80{ }^{\circ} \mathrm{C}$ in the coagulation bath. Measurements were done in a capillary tube. The corresponding 4PP resistance (blue dots) for each voltage (black squares) data point is shown on a logarithmic scale. The solid lines are an eye guide for connecting experimental data points.

Based on the signal output as a function of fiber length, area and resistance, a more detailed picture on the working principle of C4D for fibers can be seen. A rationale for the electric field coupling to the fiber mechanism can be found on Gauss laws, in relation to charge density. The charge present on the sample, $Q$, is dependent to its volume and charge density. Charge density on the fiber is that of provided by the coupling medium or dielectric material, such as the capillary tube or air. The amount of induced charge per unit time on the fiber will ultimately dictate the measured voltage. This explains fiber charging and decay for samples shorter and larger than detection gap, respectively. A representation of the following is shown in Figure 4, adapted from reference 29. For short fibers, total charge on fiber is below its maximum as fiber length is shorter than the full electrode width (A). After reaching a maximum (B), induced charges distribute along its whole length, reducing the charge density. When comparing samples equal in length, increase of fiber area will lead to higher charging, leading to higher current and, hence, to higher voltage output. The voltage increase with the fiber volume appears to follow an exponential relationship (Figure S3), until it reaches a saturation point or plateau. The amplitude of such plateau might be dependent on the fiber dimension. $(C)$ In relation to fiber charge, or effective coupling capacitance, is the resistance detection limit. As illustrated in Figure 3, and further discussed in following section, changes in resistance with values below detection limit range will be negligible respect to the high coupling impedance present in the sensor (e.g. low coupling capacitance). This sets a practical 
operating mode, where C4D can be used as an area or conductivity sensor, for fibers below or above resistance threshold.

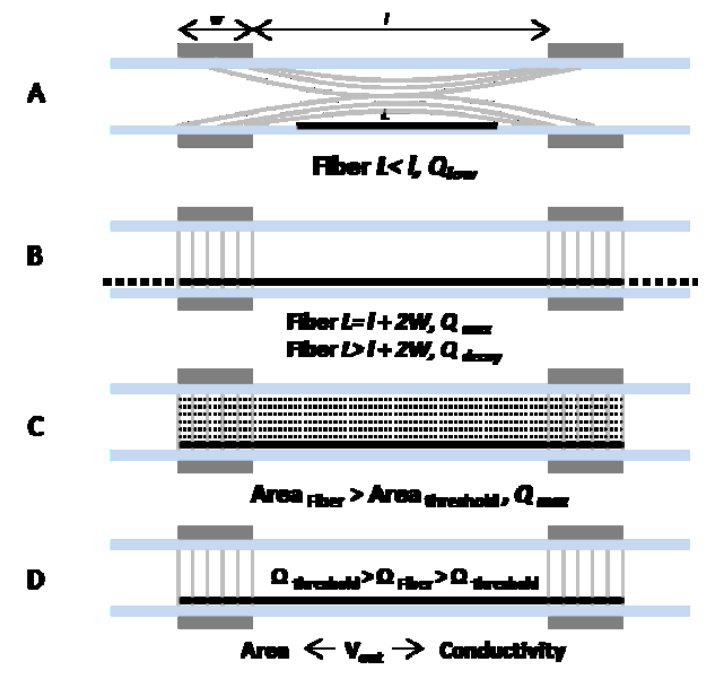

Figure 4. Schematic representation of factors influencing the C4D signal response to conductive fibers. Electrode width, w, electrode gap, I and fiber length L. A) Reduced charge density on fiber for lengths below the full electrode width. B) Maximum charge density at fiber lengths equal to full electrode width, and reduced charge density as length increases. C) Increased charge density as fibers area approaches to radial electrode area. D) Resistance detection limit defines the voltage response to changes on area, or both resistance and area.

\section{Area estimation}

Once identified the key aspects on signal output as a function of fiber properties, next and ultimate goal is to extract quantitative information on C4D measurements for fibers. Since the detector might be used using a capillary tube or air measurements, analysis on both experimental setups were carried out. It has been shown that C4D signal is highly sensitive to area variation and independent to changes in resistance - for this particular sensor and fibers studied. Without having identified yet the effective coupling capacitance, it is useful to observe the relationship between voltage and area. This is shown in Figures $5 a$ and $5 b$, with the experimental data resulting from the capillary and air measurements, respectively. Data points (black squares) are connected with a mono-exponential function (red line) described in equations 7 and 8. 

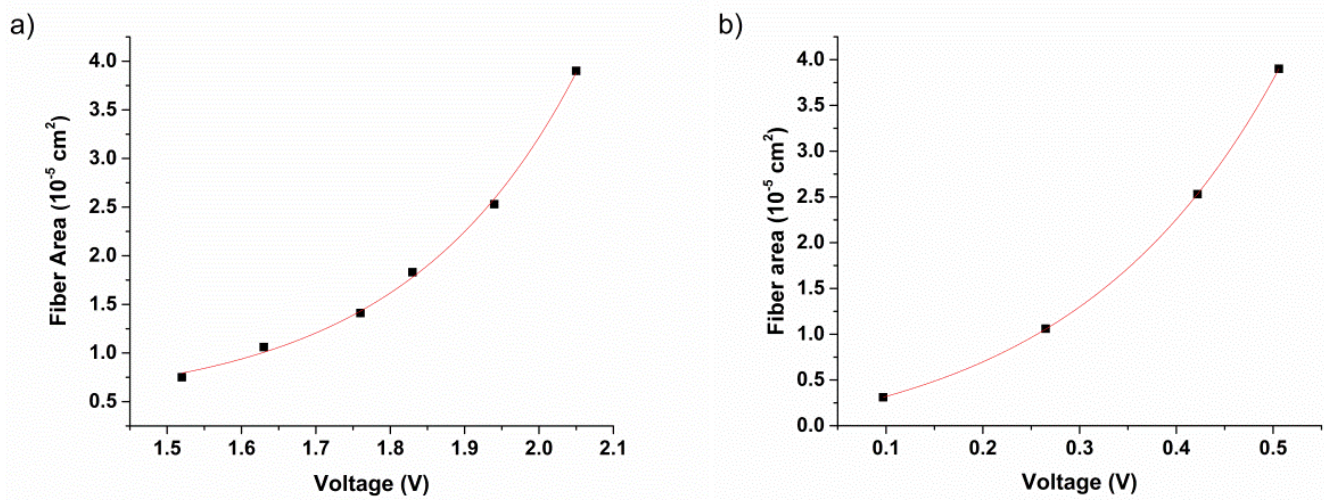

Figure 5. Experimental values of fiber area in relation to signal output (squares), and their fitting function (red line), measured in a capillary (a) and air (b). Areas expressed in $10^{-5} \mathrm{~cm}^{2}$. (a) Each data point corresponds to the voltage value listed in Table 1, for Fibers F1-F6 of $2 \mathrm{~cm}$ in length. (b) Voltage values taken from offset values of the fitting functions, listed on table S1.

In both data sets, the experimental data follow a clear exponential trend in good agreement to the fitting function $\left(R^{2}=0.99\right)$. The resulting fitting for capillary and air measurements are shown in equations 7 and 8, respectively. Areas expressed in $10^{-5} \mathrm{~cm}^{2}$ and voltage in Volts.

$$
\begin{gathered}
\text { Area }=5.2 \cdot 10^{-4} \exp \left(\frac{\text { Voltage }}{0.233}\right)+0.439 \text { (7) } \\
\text { Area }=0.398 \exp \left(\frac{\text { Voltage }}{0.214}\right)-0.314 \text { (8) }
\end{gathered}
$$

These equations serve as an empirical model for extracting the fiber's area from its voltage value, either for capillary or air measurements. Since the resistance of the studied fibers are well below the estimated resistance detection limit, voltage output is due to changes in coupling capacitance, which in turn is due to changes in fiber area. Knowing the cross-sectional area from the voltage output, and the specific conductivity $(\mathrm{S} / \mathrm{cm})$ of the test sample, it is then possible to determine the fiber conductivity. For instance for SS, of known specific resistivity $\left(8.06 \cdot 10^{-6} \mathrm{Ohms} \cdot \mathrm{cm}\right)$, and a voltage value of 0.097 , results on an estimated resistance of $25.8 \mathrm{Ohms}$ (error of $0.7 \%$ ) after introducing values in equations 8 and 5 . Same approach can be applied for RGO fibers, although the intrinsic variable resistivity of RGO fibers difficult an accurate estimation. This was seen during the preparation of the RGO fibers, where fiber conductivity was not a constant value as in metals, rather variable depending on the fiber area. A rationale for such effect is the hindered reduction of the GO component for thicker fibers respect to thinner fibers. In our case, using an average specific 
resistivity is $1.49 \cdot 10^{-2} \mathrm{Ohms} \cdot \mathrm{cm}$, results on an estimation error up to $10 \%$ respect to $4 \mathrm{PP}$ measurements.

Important to mention is that the method is independent on the fiber cross-sectional shape, in contrast to other laser based methods. It was also found that the sensor is not sensitive to the overall fiber periphery, but to the conductive regions only.

\section{Coaxial fibers}

Another possibility of C4D is to measure conductive fibers coated with dielectric materials, or also known as enamelled or coaxial fibers. In this case, and as a reference model, F3 fibers were coated with Parylene, using a molecular vapour deposition method. This resulted on a homogeneous coating and of controllable thickness. To evaluate the effect of the dielectric layer, different coating thicknesses were prepared. As shown in Figure S4 and table S2, these coatings were 6.5, 14.1, and 20.7 micron thick. The thickness was regulated by the amount of Parylene dimer used for each cycle. Measurements of coated F3 were carried out in a capillary tube, for $2 \mathrm{~cm}$ sample length. The measured voltages are listed on table S2. No marked difference in the signal amplitude could be observed, although a slightly reduced voltage output could be expected as the dielectric layer is increased. Due to the use of high excitation frequency, the dielectric layer had negligible effect on the voltage output. The differences in voltage amplitude are attributed to differences in RGO fiber area. Same as mentioned above, upon sensor calibration, the fiber area can be extracted from voltage output, and in turn, fiber conductivity can be calculated if the specific resistivity of the material is known. Alternatively, for fibers of known area and coupling capacitance, the fiber resistance underneath the dielectric layer could also be calculated.

\section{Estimation of effective coupling capacitance}

In addition to the empirical method for fiber area determination, a parametric analysis of voltage output was also performed. Since the voltage change in response to area variation is due to the increase coupling capacitance, it is reasonable to expect a proportional relation between effective coupling capacitance and area. To find out the effective coupling capacitance, further analysis of data on Figure S3 was done. Here, by applying a mono-exponential fitting function to the experimental data $\left(R^{2}=0.99\right)$, and extrapolating to large area values, a constant voltage value of $7.12 \mathrm{~V}$ was reached (see figure 6). This value corresponds to an equivalent fiber area of $5 \cdot 10^{-4} \mathrm{~cm}^{2}$. An assumption was made by attributing the limiting voltage to the sensor's geometrical capacitance. Using equation 4 , and $\varepsilon_{\mathrm{r}}$ (PTFE) $=2.1$, yields a geometrical capacitance of $0.84 \mathrm{pF}$. Upon normalisation and rescaling the fitted function to the sensor's capacitance, the coupling capacitance 
as a function of fiber area can be extracted (see figure 6 inset). For fiber F5, this yields a value of 0.19 $\mathrm{pF}$ for an area of $1.06 \cdot 10^{-5} \mathrm{~cm}^{2}$.

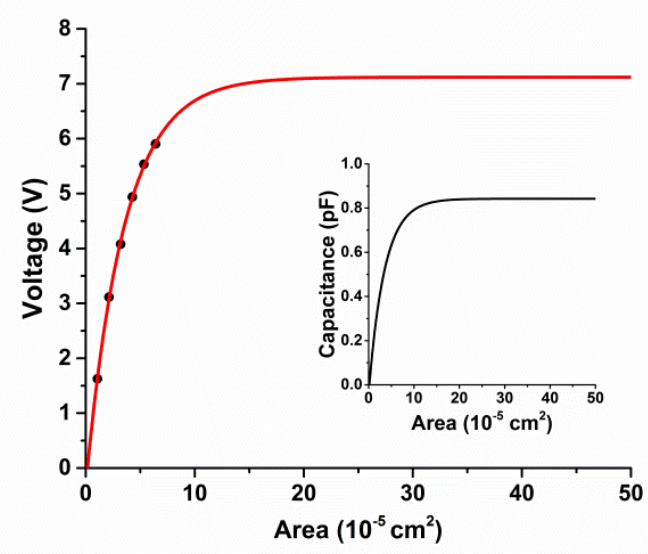

Figure 6. Voltage output as a function of $\mathrm{F} 5$ area, with experimental data points (black dots) and extrapolated values using a mono-exponential function (red line). Inset: Effective coupling capacitance of F5 as a function of fiber area based on the extrapolated function and re-scaled to the geometrical capacitance.

When performing a similar approach with other fibers, different limiting voltages where obtained (data not shown), indicating that coupling capacitances must be extracted individually for each fiber when using such approach. In order to validate the estimated coupling capacitance for fiber F5, equations 1-3 were used. Introducing in the equations the estimated coupling capacitance, a factor of 6.19 between measured and expected voltage was obtained. The origin of the factor has not been fully identified, but it may correspond to an internal signal amplification within the measuring apparatus. Based on the hypothesis that the factor is constant for all fibers measured in a capillary tube, all the measured voltages using the capillary tube where divided by the factor, and extracted the coupling capacitances. The resulting capacitance values for fibers F1-F6 are listed in table S3 and represented in figure 7 as a function of their corresponding output voltage. In addition, measured areas are also plotted as a function of their corresponding voltage. The increase in voltage output follows a linear increase in coupling capacitance, while fiber cross-section varies exponentially. A similar procedure was done for air measurements, based on voltage extrapolation to $2 \mathrm{~cm}$. For the fiber SS, of known dimensions and radial shape, the correction factor was 3.22. The difference between the factors of capillary and air measurements is attributed increased dielectric coupling of PTFE and / or the off-centre fiber alignment. 


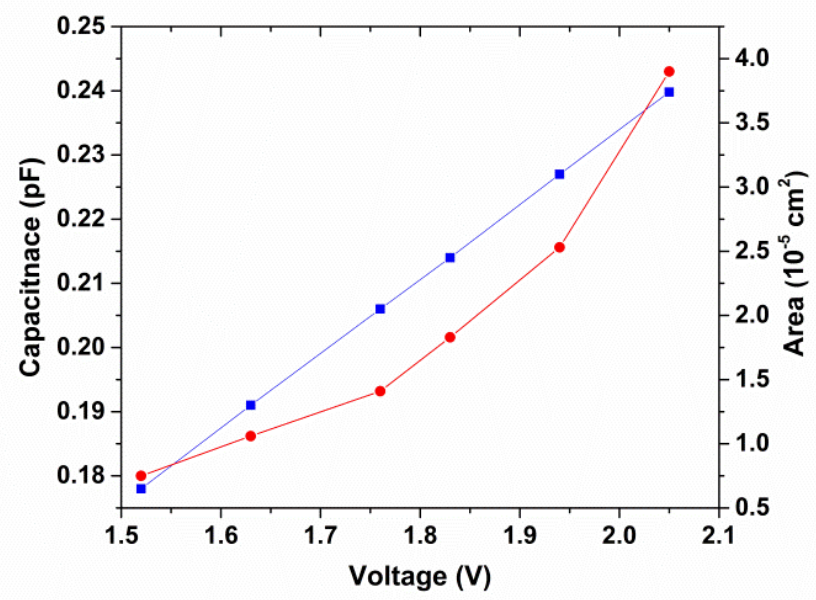

Figure 7. Estimated effective coupling capacitance (squares) and fiber area (circles) for fibers F1-F6, represented as a function of their corresponding voltage output. Voltage values are taken from measurements in capillary using fiber lengths of $2 \mathrm{~cm}$. Lines between data points are added as an eye guide.

\section{Resistance estimation and detection limit}

Last but not least, knowing the effective coupling capacitance of a single fiber, it is now possible to have a closer look to the resistance detection limit and optimum range for resistance measurements. Using equations from 1-3, and the effective coupling capacitances of fibers F1, F3, $\mathrm{F} 5$, their voltage response was simulated as a function of fiber resistance. In addition to voltage, the ratio of coupling impedance $\left(2 Z_{c}\right)$ to fiber resistance is also shown. Note that resistance values are expressed in Ohms at $1 \mathrm{~cm}$, instead of $2 \mathrm{~cm}$ as given in the calculations. The voltage values shown on the $y$-axis are converted to experimental voltages by multiplying with the factor 6.19 .

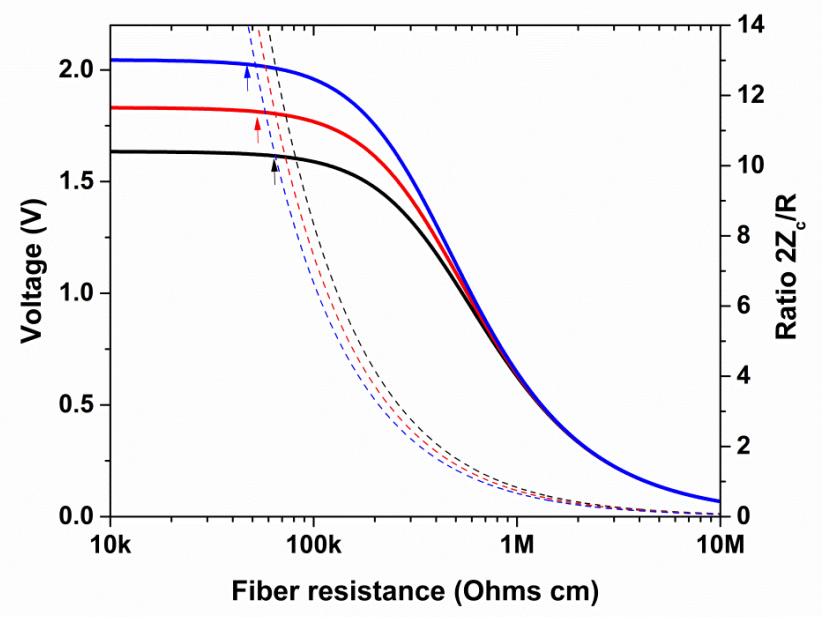


Figure 8. Simulated voltage response as a function of fiber resistance, using calculated capacitances for fibers F1 (blue), F3 (red) and F5 (black). Their respective coupling impedance to resistance ratio is also plotted as guidance. Arrows indicate resistance detection limit for each fiber.

The resistance detection limit can be seen as a voltage plateau for low resistance values, similar as in figure 3. The detection limit is chosen at the point where sensitivity is below $1 \mathrm{mV} / \mathrm{kOhm}$, corresponding to $1 \%$ change from saturation voltage. A useful term to describe the sensor response in absolute terms as function of fiber properties is the coupling impedance to resistance ratio $\left(2 Z_{c} / R\right)$. The resistance detection limit for $F 1, F 3$ and $F 5$ was very similar, of impedance ratio 14 . In terms of resistance, offset voltages correspond to 46,53 and $60 \mathrm{kOhm}$ for F1, F3 and F6, respectively. In general, the larger the area, the lower is the resistance detection limit. There is discrepancy however with the data in figure 3 in regard to the resistance detection limit, which was found experimentally at about $7 \mathrm{kOhm} \cdot \mathrm{cm}$. This is attributed to the fact that non-tensioned fibers of rough profile displayed lower voltages than expected. The increased coupling capacitance from a larger area may not be transformed into a higher voltage output if there is an air gap between electrode and fiber.

For resistance measurements using C4D, a linear range is needed. For the 3 fibers studied, the linear range is located between the 20 to $70 \%$ of the saturation voltage. In terms of $2 Z_{c} / R$, the linear range was similar for all fibers, between 2.66 and 0.63 . For example for $F 3$, this corresponds to a resistance interval from 0.28 to $1.18 \mathrm{MOhm} \cdot \mathrm{cm}$. The voltage response as function resistance had a good linear fit in such interval $\left(R^{2}=0.99\right)$, of the form: $V=a \cdot \log (R)+b$. Resistance $(R)$ in Ohms $\cdot c m$, slope $(a)$, and intercept (b). Different parameters are calculated for each fiber as listed in table S4. For air measurements, lower $Z / R$ ratios are expected for the linear range in resistance to voltage, since coupling capacitance is lower.

These results indicate that using C4D for resistance measurements can be challenging and limited to high resistance test samples. The $2 Z_{c} / R$ ratio can serve as an indicator to model the sensor for achieving the desired response (either area or resistance detector). It's is important to note that the impedance ratios are only valid for this particular sensor. Other setup with electrodes of smaller diameter will have much improved effective coupling capacitance. In general, for improving the resistance response, the $2 Z_{c} / R$ ratio should be lowered through the following variables: 1 ) increase of excitation frequency, 2) increase of electrode width, 3) large fiber area or small electrode diameter, and 4) using a capillary of higher dielectric constant and / or of reduced thickness. 


\section{Conclusions}

In summary, a thorough analysis of C4D for micron size fibers has been carried out, ranging from the fundamental working principles to empirical, parametric analysis and simulation models. Due to the coupling principle between electrodes and fiber, the sensor is highly sensitive to fiber area and length. This implies that for comparative purposes, only fibers of same length should be used. On the other hand, it was also observed that the effect of length becomes independent for long fibers. Analysis of experimental data and simulated voltage output for the different fibers investigated revealed that C4D can work as an area or resistance detector. In addition, resistance measurements are influenced by the fiber area through the coupling impedance. Therefore, only one variable can be measured at the time. The functioning mode can be categorized in absolute terms by the coupling impedance to fiber resistance ratio. Area or resistance detection occurs at high or low impedance ratios, respectively. For area determination, the sensor must be calibrated due to the non-linear relationship between area and voltage. Upon calibration, voltage response was found to be highly sensitive and independent to fiber geometry. Resistance measurements are challenging for low resistance samples, but possible with an improved sensor design and use of thicker fibers. Due to the contactless nature of the measurement, C4D could also be used to determine the electrical properties of fibers with insulating coatings, otherwise inaccessible by contact methods. This study

serves as a baseline for the design and calibration of C4D for the evaluation conductive fibers by contactless means. Applications of this approach could be beneficial for reel to reel fiber processing, or in-situ monitoring of fiber properties during additive manufacturing. Further studies on fiber characterisation could be aimed to improve sensor's resistance sensitivity, and the sensor's response to a running fiber using the air measurement setup.

\section{Acknowledgements}

This work has been supported by the Australian Research Council Centre of Excellence Scheme (Project Number CE 140100012). The authors would like to thank the Australian National Nanofabrication Facility Materials Node for equipment use.

\section{References}

1 F. Meng, W. Lu, Q. Li, J.-H. Byun, Y. Oh and T.-W. Chou, Adv. Mater., 2015, 27, 5113-5131.

2 E. Frank, L. M. Steudle, D. Ingildeev, J. M. Sporl and M. R. Buchmeiser, Angew. Chem., Int. Ed., 2014, 53, 5262-98. 
3 J. Foroughi, G. M. Spinks, D. Antiohos, A. Mirabedini, S. Gambhir, G. G. Wallace, S. R. Ghorbani, G. Peleckis, M. E. Kozlov, M. D. Lima and R. H. Baughman, Adv. Funct. Mater., 2014, 24, 5859-5865.

4 J. Foroughi, G. M. Spinks, S. R. Ghorbani, M. E. Kozlov, F. Safaei, G. Peleckis, G. G. Wallace and R. H. Baughman, Nanoscale, 2012, 4, 940-5.

5 J. Foroughi, G. M. Spinks, S. Aziz, A. Mirabedini, A. Jeiranikhameneh, G. G. Wallace, M. E. Kozlov and R. H. Baughman, ACS Nano, 2016, 10, 9129-9135.

6 M. D. Lima, N. Li, M. Jung de Andrade, S. Fang, J. Oh, G. M. Spinks, M. E. Kozlov, C. S. Haines, D. Suh, J. Foroughi, S. J. Kim, Y. Chen, T. Ware, M. K. Shin, L. D. Machado, A. F. Fonseca, J. D. W. Madden, W. E. Voit, D. S. Galvão and R. H. Baughman, Science, 2012, 338, 928-932.

7 J. Foroughi, G. M. Spinks, G. G. Wallace, J. Oh, M. E. Kozlov, S. Fang, T. Mirfakhrai, J. D. W. Madden, M. Shin, S. J. Kim and R. H. Baughman, Science, 2011, 334, 494-497.

8 W. Zeng, L. Shu, Q. Li, S. Chen, F. Wang and X. M. Tao, Adv. Mater., 2014, 26, 5310-36.

9 H. Cheng, C. Hu, Y. Zhao and L. Qu, NPG Asia Materials, 2014, 6, e113.

10 Z. Xu and C. Gao, Materials Today, 2015, 18, 480-492.

11 L. Chen, Y. Liu, Y. Zhao, N. Chen and L. Qu, Nanotechnology, 2016, 27, 032001.

12 T. Bashir, L. Fast, M. Skrifvars and N.-K. Persson, J. App. Poly. Sci., 2012, 124, 2954-2961.

13 Y. H. Ding, P. Zhang, Q. Zhuo, H. M. Ren, Z. M. Yang and Y. Jiang, Nanotechnology, 2011, 22, 215601.

14 R. Trusovas, K. Ratautas, G. Račiukaitis, J. Barkauskas, I. Stankevičienė, G. Niaura and R. Mažeikienè, Carbon, 2013, 52, 574-582.

15 S. Eigler, S. Grimm, M. Enzelberger-Heim, P. Muller and A. Hirsch, Chem. Commun., 2013, 49, 7391-3.

16 C. Mattevi, G. Eda, S. Agnoli, S. Miller, K. A. Mkhoyan, O. Celik, D. Mastrogiovanni, G. Granozzi, E. Garfunkel and M. Chhowalla, Adv. Funct. Mater., 2009, 19, 2577-2583.

17 R. Jalili, S. H. Aboutalebi, D. Esrafilzadeh, R. L. Shepherd, J. Chen, S. Aminorroaya-Yamini, K. Konstantinov, A. I. Minett, J. M. Razal and G. G. Wallace, Adv. Funct. Mater., 2013, 23, 53455354.

18 A. M. Azzeer, L. M. Silber, I. L. Spain, C. E. Patton and H. A. Goldberg, J. Appl. Phys., 1985, 57, 2529.

19 T. Bernardino, P. Antoranz, J. M. Miranda and J. L. Sebastian, European Microwave Integrated Circuit Conference,2007, 603-606.

20 N. D. Orloff, C. J. Long, J. Obrzut, L. Maillaud, F. Mirri, T. P. Kole, R. D. McMichael, M. Pasquali, S. J. Stranick and J. A. Liddle, Sci. Rep., 2015, 5, 17019. 
21 N. D. Orloff, J. Obrzut, C. J. Long, T. Lam, P. Kabos, D. R. Novotny, J. C. Booth and J. A. Liddle, IEEE Trans. Microw. Theory Techn., 2014, 62, 2149-2159.

22 J. M. Cabot, E. Duffy, S. Currivan, A. Ruland, R. Jalili, A. J. Mozer, P. C. Innis, G. G. Wallace, M. Breadmore and B. Paull, Analyst, 2016, 141, 2774-82.

23 P. Kuban and P. C. Hauser, Electrophoresis, 2004, 25, 3387-97.

24 P. Kuban and P. C. Hauser, Electrophoresis, 2004, 25, 3398-405.

25 F. Opekar, P. Tuma and K. Stulik, sensors, 2013, 13, 2786-801.

26 A. J. Zemann, E. Schnell, D. Volgger and G. K. Bonn, Anal. Chem., 1998, 70, 563-7.

27 J. A. F. da Silva and C. L. do Lago, Anal. Chem., 1998, 70, 4339-4343.

28 P. Kuban, P. C. Hauser, Electrophoresis, 2017, 38, 95-114.

29 Z. Zhenli, L. Yaolong, X. Zhongshi, Z. Xilei, K. Qi, S. Dazhong, Int. J. Electrochem. Sci., 2013, 8, 3357-3370. 


\section{Electronic Supporting information for:}

\section{Quantitative Characterisation of Conductive Fibers by Capacitive Coupling}

Andres Ruland, Rouhollah Jalili, Attila J. Mozer and Gordon G. Wallace

ARC Centre of Excellence for Electromaterials Science (ACES), Intelligent Polymer Research Institute, AlIM Facility, Innovation Campus, University of Wollongong, NSW 2500, Australia.
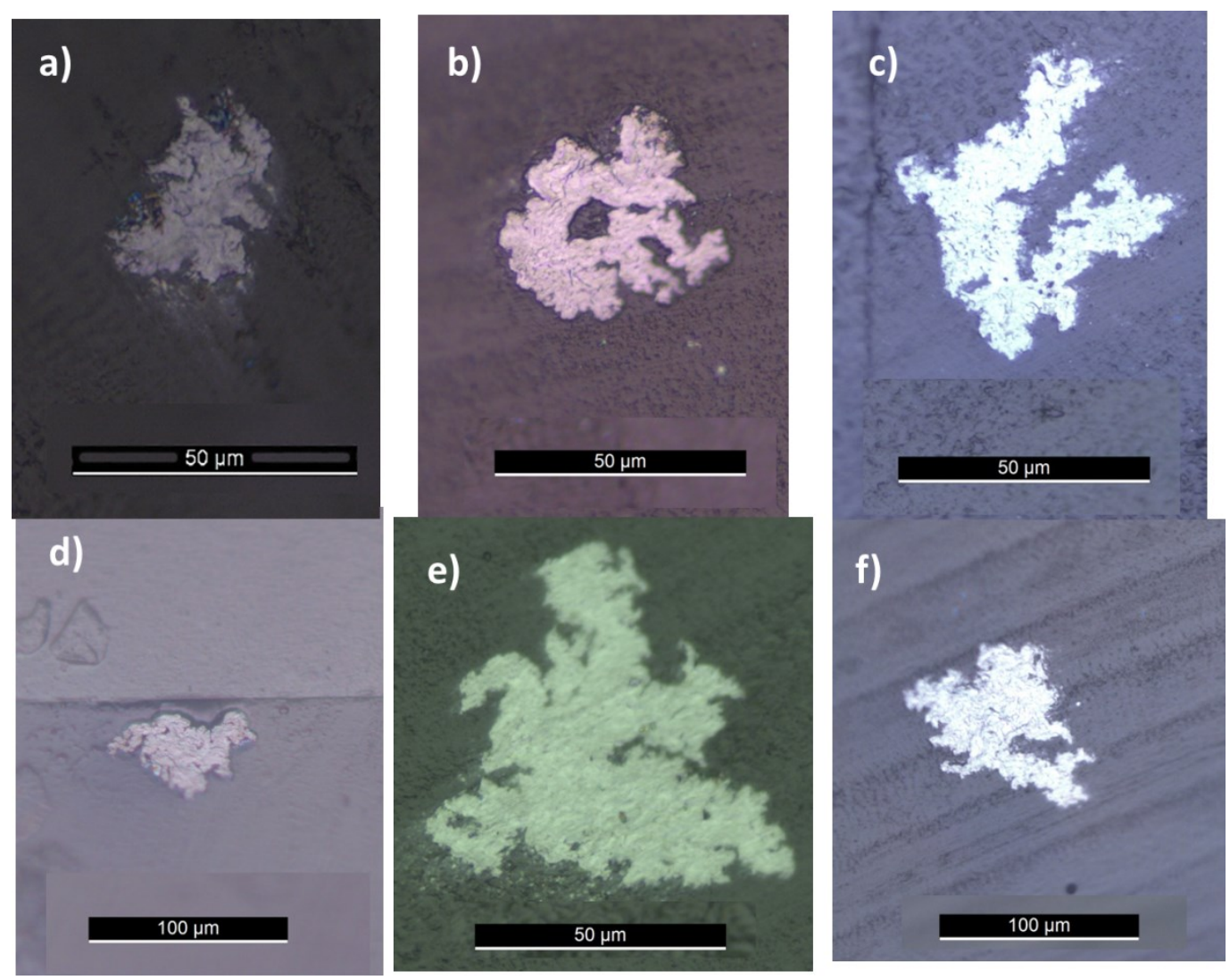

Figure S1. Optical microscope photographs displaying cross-sectional images of the RGO used in this study. From smaller to larger cross-section areas; a) F6, b)F5, c)F4, d)F3, e)F2, f)F1. 
a)

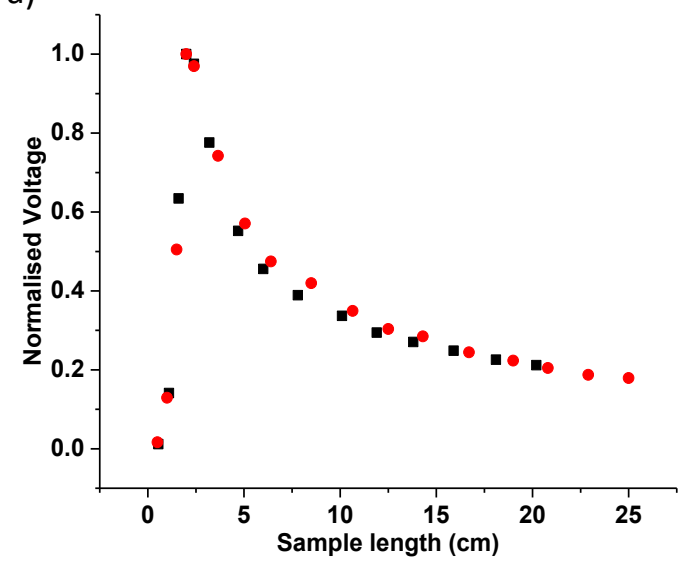

b)

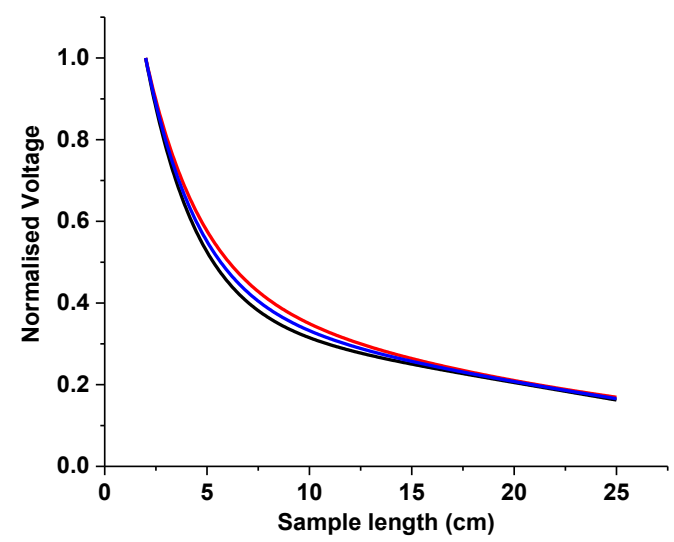

Figure S2. (a) Normalised comparison voltage output of F2 (circles) and F5 (squares). (b) Comparison of the fitting functions for F2 (red), F5 (black) and their average (blue). The bi-exponential fitting function used was: $y=A_{1} \cdot \exp \left(-x / t_{1}\right)+A_{2} \cdot \exp \left(-x / t_{2}\right)+y_{0}$

Table S1. Extracted fitting coefficients from fiber length dependence done in air measurements.

\begin{tabular}{|c|c|c|c|c|}
\hline & F1 & F2 & F5 & SS \\
\hline y0 & 0.506 & 0.420 & 0.265 & 0.097 \\
\hline A1 & $2.654(59 \%)$ & $0.992(43 \%)$ & $2.101(62 \%)$ & $0.916(59 \%)$ \\
\hline T1 & 3.596 & 7.798 & 4.310 & 8.397 \\
\hline A2 & $1.773(41 \%)$ & $1.294(57 \%)$ & $1.301(38 \%)$ & $0.647(41 \%)$ \\
\hline T2 & 21.429 & 25.770 & 22.663 & 49.564 \\
\hline
\end{tabular}

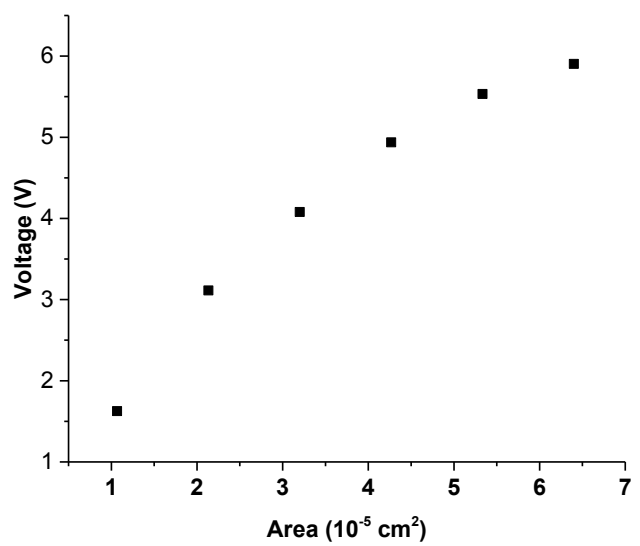

Figure S3. Voltage output as a function of F5 area. X-axis is recalculated from the number of fiber fragments based on a fiber of cross-sectional area of $1.06 \cdot 10^{-5} \mathrm{~cm}^{2}$. 


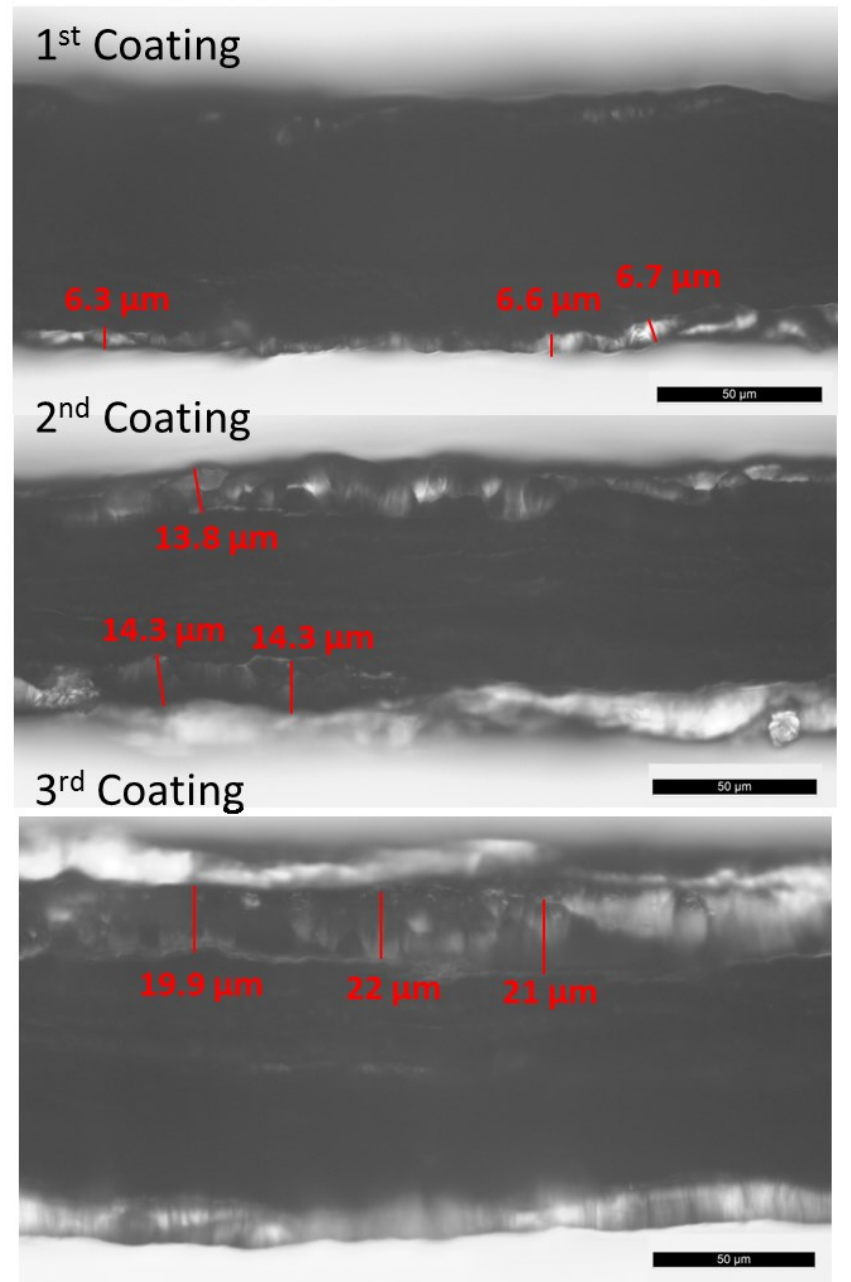

Figure S4. Optical microscope images of lateral sections from Parylene coated RGO fibers, batch F3, resulting from the different coating cycles.

Table S2. Summary of Parylene coated fibers, with the C4D voltage output as a function of coating cycle.

\begin{tabular}{|c|c|c|}
\hline Coating Cycle & $\begin{array}{c}\text { Parylene } \\
\text { thickness }(\boldsymbol{\mu m})\end{array}$ & $\begin{array}{c}\text { Voltage (V) } \\
\text { at } \mathbf{2} \mathbf{~ M H z}\end{array}$ \\
\hline 0 & 0 & 1.83 \\
\hline 1 & 6.3 & 1.83 \\
\hline 2 & 14.3 & 1.85 \\
\hline 3 & 20.9 & 1.84 \\
\hline
\end{tabular}


Table S3. List of measured voltages (exp.) and estimated capacitances derived from the factor extracted from fiber F5.

\begin{tabular}{|c|c|c|}
\hline Sample & $\begin{array}{c}\text { Voltage } \\
\text { (exp.) }\end{array}$ & $\begin{array}{c}\mathbf{C}_{\mathbf{c}} \\
\text { (factor) }\end{array}$ \\
\hline F1 & 2.05 & 0.239 \\
\hline F2 & 1.94 & 0.227 \\
\hline F3 & 1.83 & 0.214 \\
\hline F4 & 1.76 & 0.206 \\
\hline F5 & 1.63 & 0.191 \\
\hline F6 & 1.52 & 0.178 \\
\hline
\end{tabular}

Table S4. Regression coefficients for voltage output as a function of fiber resistance. $V=a \cdot \log (R)+b, R$ in Ohms $\cdot \mathrm{cm}$.

\begin{tabular}{|c|c|c|}
\hline Fiber & a & b \\
\hline F1 & -1.677 & 10.693 \\
\hline F2 & -1.50745 & 9.68143 \\
\hline F6 & -1.345 & 8.706 \\
\hline
\end{tabular}

